\title{
PERCOLATION IN THE HYPERBOLIC PLANE
}

\author{
ITAI BENJAMINI AND ODED SCHRAMM
}

\section{INTRODUCTION}

The purpose of this paper is to study percolation in the hyperbolic plane and in transitive planar graphs that are quasi-isometric to the hyperbolic plane.

There are several sources available which the reader may consult for background on percolation on $\mathbb{Z}^{d}[\mathrm{Gri} 89]$ and $\mathbb{R}^{d}$ [MR96] and for background on percolation on more general graphs [BS96], [Lyo00], [BS99]. For this reason, we will be quite brief here. Background on hyperbolic geometry may be found in [CFKP97] and the references sited there.

Percolation on planar hyperbolic graphs. A graph $G$ is transitive if the automorphism group of $G$ acts transitively on the vertices $\mathrm{V}(G)$. An invariant percolation on a graph $G$ is a probability measure on the space of subgraphs of $G$, which is invariant under the automorphisms of $G$. The connected components of the random subgraph are often called clusters. Of special interest are the Bernoulli site and bond percolation. The Bernoulli $(p)$ bond percolation is the random subgraph with vertices $\mathrm{V}(G)$, and where each edge is in the percolation subgraph with probability $p$, independently. In Bernoulli $(p)$ site percolation, each vertex is in the percolation subgraph with probability $p$, independently, and an edge appears in the percolation subgraph iff its endpoints are present.

Let $G$ be an infinite, connected, planar, transitive graph, with finite vertex degree. Each such graph is quasi-isometric with one and only one of the following spaces: $\mathbb{Z}$, the 3 -regular tree, the Euclidean plane $\mathbb{R}^{2}$, and the hyperbolic plane $\mathbb{H}^{2}$ [Bab97]. Each of these classes has its distinct geometry, and random processes behave similarly on graphs within each class. On trees, Bernoulli percolation is quite simple, but there are some interesting results concerning invariant percolation [Häg97]. Bernoulli percolation on $\mathbb{Z}^{2}$ and planar lattices in $\mathbb{R}^{2}$ has an extensive theory. (See [Gri89].) Burton and Keane [BK91] also obtained a theory of invariant percolation in $\mathbb{Z}^{2}$.

A transitive graph $G$ has one end if for every finite set of vertices $V_{0} \subset \mathrm{V}(G)$ there is precisely one infinite connected component of $G \backslash V_{0}$. A transitive planar graph $G$ with one end is quasi-isometric to $\mathbb{R}^{2}$ or to $\mathbb{H}^{2}$ (see, e.g., [Bab97] or the proof of Proposition 2.1(b), below). Amenability is a simple geometric property which distinguishes these two possibilities. $G$ is amenable if for every $\epsilon>0$ there

Received by the editors January 18, 2000 and, in revised form, November 9, 2000.

2000 Mathematics Subject Classification. Primary 82B43; Secondary 60K35, 60D05.

Key words and phrases. Poisson process, Voronoi, isoperimetric constant, nonamenable, planarity, Euler formula, Gauss-Bonnet theorem.

The second author's research was partially supported by the Sam and Ayala Zacks Professorial Chair at the Weizmann Institute. 
is a finite set of vertices $V_{0}$ such that $\left|\partial V_{0}\right|<\epsilon\left|V_{0}\right|$. A graph quasi-isometric with $\mathbb{H}^{2}$ must be nonamenable. Grimmett and Newman [GN90] have shown that for some parameter values, Bernoulli percolation on the cartesian product of $\mathbb{Z}$ with a regular tree of sufficiently high degree has infinitely many infinite components. This cannot happen on amenable graphs, by the Burton-Keane argument [BK89].

Let $p_{u}=p_{u}(G)$ be the infimum of the set of $p \in[0,1]$ such that $\operatorname{Bernoulli}(p)$ percolation on $G$ has a unique infinite cluster a.s. The critical parameter $p_{c}=p_{c}(G)$ is defined as the infimum of the set of $p \in[0,1]$ such that $\operatorname{Bernoulli}(p)$ percolation on $G$ has an infinite cluster a.s. It has been conjectured [BS96] that $p_{c}(G)<p_{u}(G)$ for every nonamenable transitive graph $G$. It has been recently proven by Pak and Smirnova-Nagnibeda [PSN00] that every nonamenable group has some Cayley graph $G$ satisfying $p_{c}(G)<p_{u}(G)$. Lalley [Lal98] proved $p_{c}(G)<p_{u}(G)$ for planar Cayley graphs of Fuchsian groups with sufficiently high genus. We now show

Theorem 1.1. Let $G$ be a transitive, nonamenable, planar graph with one end. Then $0<p_{c}(G)<p_{u}(G)<1$, for Bernoulli bond or site percolation on $G$.

The hyperbolic plane seems to be a very good testing ground for conjectures about nonamenable graphs. The planarity and hyperbolic geometry help to settle questions that may be more difficult in general.

Theorem 1.2. Let $G$ be a transitive, nonamenable, planar graph with one end. Then Bernoulli $\left(p_{u}\right)$ percolation on $G$ has a unique infinite cluster a.s.

This contrasts with a result of Schonmann [Sch99a], which shows that a.s. the number of infinite components of $\operatorname{Bernoulli}\left(p_{u}\right)$ percolation on $T \times \mathbb{Z}$ is either 0 or $\infty$ when $T$ is a regular tree. (If $T$ is a regular tree of sufficiently high degree, then $p_{u}(T \times \mathbb{Z})>p_{c}(T \times \mathbb{Z})$, by [GN90], and hence there are infinitely many infinite components at $p_{u}$ on $T \times \mathbb{Z}$.) Peres [Per00] has generalized this result of [Sch99a] (with a different proof) to nonamenable products.

It is known that on a transitive graph $G$ when $p>p_{u}(G)$, Bernoulli $(p)$ percolation a.s. has a unique infinite cluster. This has been proven by Häggström and Peres [HP99] in the unimodular setting, and in full generality by Schonmann [Sch99b]. The proof of Theorem 1.2 will also prove this "uniqueness monotonicity" in this restricted setting.

The situation at $p_{c}$ is also known. The following theorem is from [BLPS99a]; see also [BLPS99b].

Theorem 1.3. Let $G$ be a nonamenable graph with a vertex-transitive unimodular automorphism group. Then a.s. critical Bernoulli bond or site percolation on $G$ has no infinite components.

Note that a planar transitive graph with one end has a unimodular automorphism group (Proposition 2.1). Hence the above theorem applies to the graphs under consideration here.

Percolation in $\mathbb{H}^{2}$. Though percolation is usually studied on graphs, and many interesting phenomena already appear in the graph setup, there are some special properties that only appear in the continuous setting.

We therefore consider the Poisson-Voronoi-Bernoulli percolation model in the hyperbolic plane $\mathbb{H}^{2}$. There are two parameters needed to specify the model, $\lambda>0$ and $p \in[0,1]$. Given such a pair $(p, \lambda)$, consider a Poisson point process with intensity $\lambda$ in the hyperbolic plane. Such a process gives rise to a Voronoi tessallation of 


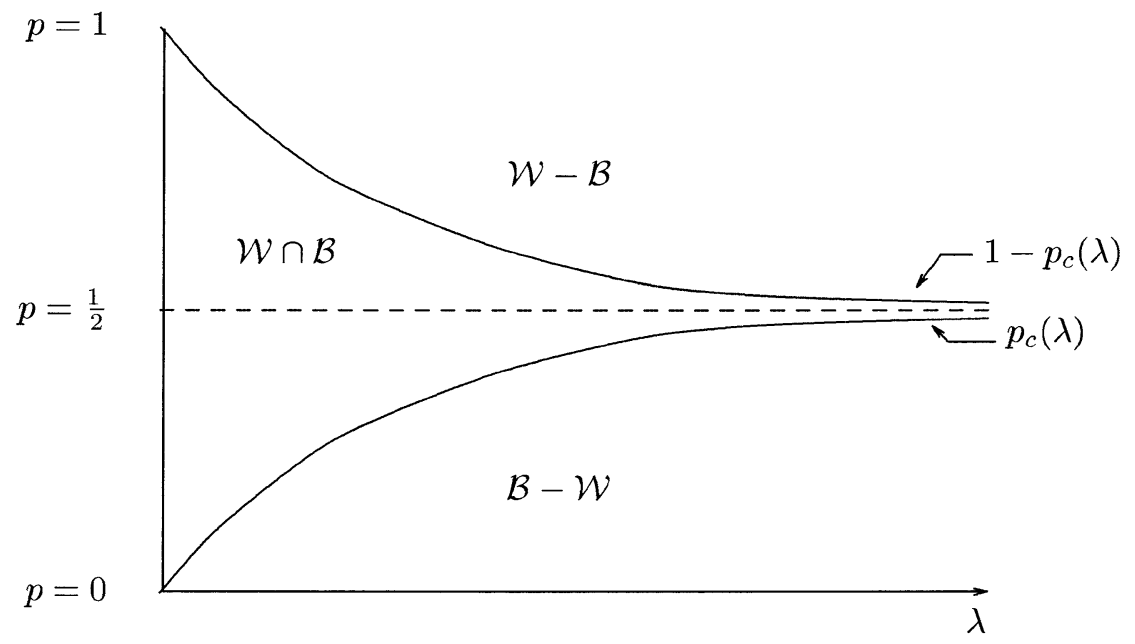

Figure 1.1. The phase diagram of Voronoi percolation

$\mathbb{H}^{2}$. Each tile of this tessallation is colored white (respectively, black) with probability $p$ (respectively, $1-p$ ), independently. The union of all the white (resp. black) tiles is denoted by $\widehat{W}$ (resp. $\widehat{B})$. Let $\mathcal{W}($ resp. $\mathcal{B})$ denote the set of pairs $(p, \lambda)$ such that $\widehat{W}$ (resp. $\widehat{B})$ has an unbounded component a.s. For $\lambda>0$, let

$$
p_{c}(\lambda):=\inf \{p \in[0,1]:(p, \lambda) \in \mathcal{W}\} .
$$

We prove the following (see Figure 1.1)

Theorem 1.4. Consider $(p, \lambda)$-Voronoi percolation in $\mathbb{H}^{2}$.

(a) If $(p, \lambda) \in \mathcal{W} \cap \mathcal{B}$, then there are a.s. infinitely many unbounded components of $\widehat{W}$ and there are infinitely many unbounded components of $\widehat{B}$.

(b) If $(p, \lambda) \in \mathcal{W} \backslash \mathcal{B}$, then a.s. there is a unique unbounded components of $\widehat{W}$, and no unbounded components of $\widehat{B}$.

(c) If $(p, \lambda) \in \mathcal{B} \backslash \mathcal{W}$, then a.s. there is a unique unbounded component of $\widehat{B}$, and no unbounded components of $\widehat{W}$.

Note that, by symmetry,

$$
\mathcal{B}=\{(p, \lambda):(1-p, \lambda) \in \mathcal{W}\}
$$

Theorem 1.5. (a) $0<p_{c}(\lambda) \leq \frac{1}{2}-\frac{1}{4 \lambda \pi+2}<\frac{1}{2}$.

(b) $\lim _{\lambda \rightarrow 0} p_{c}(\lambda)=0$.

(c) $p_{c}(\lambda)$ is continuous.

(d) $\left(p_{c}(\lambda), \lambda\right) \notin \mathcal{W}$ for all $\lambda>0$, and hence $\mathcal{W}=\left\{(p, \lambda): p>p_{c}(\lambda)\right\}$.

We conjecture that $p_{c}(\lambda) \rightarrow 1 / 2$ as $\lambda \rightarrow \infty$. Note that percolation with parameters $(p, \lambda)$ on $\mathbb{H}^{2}$ is equivalent to percolation with parameters $(p, 1)$ on $\mathbb{H}^{2}$ with the metric rescaled by $\sqrt{\lambda}$. Hence, taking $\lambda \rightarrow \infty$ amounts to the same as letting the curvature tend to zero. This means that Voronoi percolation on $\mathbb{R}^{2}$ can be seen as a limit of Voronoi percolation on $\mathbb{H}^{2}$. See Question 7.5 for further discussion of this issue. 
We generalize the Mass Transport Principle ([Häg97], [BLPS99a]) to the hyperbolic plane, and use it as a tool for our investigations. One consequence of the Mass Transport Principle that we derive and use is a generalization of Euler's formula $|V|-|E|+|F|=2$ relating the number of vertices, edges, and faces in a (finite) tiling of the sphere to random (infinite) tilings of the hyperbolic plane with invariant law.

A collection of open problems is presented at the end of the paper. Most of these are related to the Voronoi percolation model. We believe that this process deserves further study.

\section{TERminology AND PRELIMINARIES}

All the graphs that we shall consider in this paper are locally finite; that is, each vertex has finitely many incident edges. The vertices of a graph $G$ will be denoted by $\mathrm{V}(G)$, and the edges by $\mathrm{E}(G)$.

Given a graph $G$, let $\operatorname{Aut}(G)$ denote the group of automorphisms of $G$. $G$ is transitive if $\operatorname{Aut}(G)$ acts transitively on the vertices $\mathrm{V}(G)$. $G$ is quasi-transitive if $\mathrm{V}(G) / \operatorname{Aut}(G)$ is finite; that is, there are finitely many $\operatorname{Aut}(G)$ orbits in $\mathrm{V}(G)$. A graph $G$ is unimodular if $\operatorname{Aut}(G)$ is a unimodular group (which means that the left-invariant Haar measure is also right-invariant). Cayley graphs are unimodular, and any graph such that $\operatorname{Aut}(G)$ is discrete is unimodular. See [BLPS99a] for a further discussion of unimodularity and its relevance to percolation.

An invariant percolation on $G$ is a probability measure on the space of subgraphs of $G$, which is $\operatorname{Aut}(G)$-invariant. A cluster is a connected component of the percolation subgraph.

Let $X=\mathbb{R}^{2}$ or $X=\mathbb{H}^{2}$. We say that an embedded graph $G \subset X$ in $X$ is properly embedded if every compact subset of $X$ contains finitely many vertices of $G$ and intersects finitely many edges. Suppose that $G$ is an infinite connected graph with one end, properly embedded in $X$. Let $G^{\dagger}$ denote the dual graph of $G$. We assume that $G^{\dagger}$ is embedded in $X$ in the standard way relative to $G$; that is, every vertex $v^{\dagger}$ of $G^{\dagger}$ lies in the corresponding face of $G$, and every edge $e \in \mathrm{E}(G)$ intersects only the dual edge $e^{\dagger} \in \mathrm{E}\left(G^{\dagger}\right)$, and only in one point. If $\omega$ is a subset of the edges $\mathrm{E}(G)$, then $\omega^{\dagger}$ will denote the set

$$
\omega^{\dagger}:=\left\{e^{\dagger}: e \notin \omega\right\} .
$$

Given $p \in[0,1]$ and a graph $G$, we often denote the percolation graph of $\operatorname{Bernoulli}(p)$ bond percolation on $G$ by $\omega_{p}$.

Proposition 2.1. Let $G$ be a transitive, nonamenable, planar graph with one end, and let $\Gamma$ be the group of automorphisms of $G$.

(a) $\Gamma$ is discrete (and hence unimodular).

(b) $G$ can be embedded as a graph $G^{\prime}$ in the hyperbolic plane $\mathbb{H}^{2}$ in such a way that the action of $\Gamma$ on $G^{\prime}$ extends to an isometric action on $\mathbb{H}^{2}$. Moreover, the embedding can be chosen in such a way that the edges of $G^{\prime}$ are hyperbolic line segments.

A sketch of the proof of (b) appears in [Bab97]. We include a proof here, for completeness.

Proof of Proposition 2.1. By $[\operatorname{Mad} 70]$ or by [Wat70] it follows that $G$ is 3-vertex connected; that is, every finite nonempty set of vertices $V_{0} \subset \mathrm{V}(G), V_{0} \neq \varnothing$, neighbors with at least 3 vertices in $\mathrm{V}(G) \backslash V_{0}$. Therefore, by the extension of Imrich 
to Whitney's Theorem [Imr75], the embedding of $G$ in the plane is unique, in the sense that in any two embeddings of $G$ in the plane, the cyclic orientation of the edges going out of the vertices is either identical for all the vertices, or reversed for all the vertices. This implies that an automorphism of $G$ that fixes a vertex and all its neighbors is the identity, and therefore $\operatorname{Aut}(G)$ is discrete. For a discrete group, the counting measure is Haar measure, and is both left- and right-invariant. Hence $\operatorname{Aut}(G)$ is unimodular. This proves part (a).

Think of $G$ as embedded in the plane. Call a component of $S^{2}-G$ a face if its boundary consists of finitely many edges in $G$. In each face $f$ put a new vertex $v_{f}$, and connect it by edges to the vertices on the boundary of $f$. If this is done appropriately, then the resulting graph $\widehat{G}$ is still embedded in the plane. Note that $\widehat{G}$ together with all its faces forms a triangulation $T$ of a simply connected domain in $S^{2}$. To prove (b) it is enough to produce a triangulation $T^{\prime}$ of $\mathbb{H}^{2}$ isomorphic with $T$ such that the elements of $\operatorname{Aut}\left(T^{\prime}\right)$ extend to isometries of $\mathbb{H}^{2}$ and the edges of $T^{\prime}$ are hyperbolic line segments. There are various ways to do this; one of them is with circle packing theory. See, for example, [BSt90], [HS95], or [Bab97].

\section{The number of COMPonents}

Theorem 3.1. Let $G$ be a transitive, nonamenable, planar graph with one end, and let $\omega$ be an invariant bond percolation on $G$. Let $k$ be the number of infinite components of $\omega$, and let $k^{\dagger}$ be the number of infinite components of $\omega^{\dagger}$. Then a.s.

$$
\left(k, k^{\dagger}\right) \in\{(1,0),(0,1),(1, \infty),(\infty, 1),(\infty, \infty)\} .
$$

Remark 3.2. Each of these possibilities can happen. The case $\left(k, k^{\dagger}\right)=(1, \infty)$ appears when $\omega$ is the free spanning forest of $G$, while $(\infty, 1)$ is the situation for the wired spanning forest. See [BLPS00]. The other possibilities occur for Bernoulli percolation, as we shall see.

Lemma 3.3. Let $G$ be a transitive, nonamenable, planar graph with one end. Let $\omega$ be an invariant percolation on $G$. If $\omega$ has only finite components a.s., then $\omega^{\dagger}$ has infinite components a.s.

The proof will use a result from [BLPS99a], which says that when the expected degree $\mathbf{E} \operatorname{deg}_{\omega} v$ of a vertex $v$ in an invariant percolation on a unimodular nonamenable graph $G$ is sufficiently close to $\operatorname{deg}_{G} v$, there are infinite clusters in $\omega$ with positive probability. (The case of trees was established earlier in [Häg97].)

Proof. Suppose that both $\omega$ and $\omega^{\dagger}$ have only finite components a.s. Then a.s. given a component $K$ of $\omega$, there is a unique component $K^{\prime}$ of $\omega^{\dagger}$ that surrounds it. Similarly, for every component $K$ of $\omega^{\dagger}$, there is a unique component $K^{\prime}$ of $\omega$ that surrounds it. Let $\mathcal{K}_{0}$ denote the set of all components of $\omega$. Inductively, set

$$
\mathcal{K}_{j+1}:=\left\{K^{\prime \prime}: K \in \mathcal{K}_{j}\right\} .
$$

For $K \in \mathcal{K}_{0}$ let $r(K):=\sup \left\{j: K \in \mathcal{K}_{j}\right\}$ be the rank of $K$, and define $r(v):=r(K)$ if $K$ is the component of $v$ in $\omega$. For each $r$ let $\omega^{r}$ be the set of edges in $\mathrm{E}(G)$ incident with vertices $v \in \mathrm{V}(G)$ with $r(v) \leq r$. Then $\omega^{r}$ is an invariant bond percolation and

$$
\lim _{r \rightarrow \infty} \mathbf{E}\left[\operatorname{deg}_{\omega^{r}} v\right]=\operatorname{deg}_{G} v
$$


Consequently, by the above result from [BLPS99a], we find that $\omega^{r}$ has with positive probability infinite components for all sufficiently large $r$. This contradicts the assumption that $\omega$ and $\omega^{\dagger}$ have only finite components a.s.

The following has been proven in [BLPS99a] and [BLPS99b] in the transitive case. The extension to the quasi-transitive case is straightforward.

Theorem 3.4. Let $G$ be a nonamenable, quasi-transitive, unimodular graph, and let $\omega$ be an invariant percolation on $G$ which has a single component a.s. Then $p_{c}(\omega)<1$ a.s.

The following has been proven in [BLPS99a].

Lemma 3.5. Let $G$ be a quasi-transitive, nonamenable, planar graph with one end, and let $\omega$ be an invariant percolation on $G$. Then a.s. the number of infinite components of $\omega$ is 0,1 , or $\infty$.

For the sake of completeness, we present a (somewhat different) proof here.

Proof. In order to reach a contradiction, assume that with positive probability $\omega$ has a finite number $k>1$ of infinite components, and condition on that event. Select at random, uniformly, a pair of distinct infinite components $\omega_{1}, \omega_{2}$ of $\omega$. Let $\omega_{1}^{c}$ be the subgraph of $G$ spanned by the vertices outside of $\omega_{1}$, and let $\tau$ be the set of edges of $G$ that connect vertices in $\omega_{1}$ to vertices in the component of $\omega_{1}^{c}$ containing $\omega_{2}$. Set

$$
\tau^{\dagger}:=\left\{e^{\dagger}: e \in \tau\right\}
$$

Then $\tau^{\dagger}$ is an invariant bond percolation in the dual graph $G^{\dagger}$. Using planarity, it is easy to verify that $\tau^{\dagger}$ is a.s. a bi-infinite path. This contradicts Theorem 3.4, and thereby completes the proof.

Corollary 3.6. Let $G$ be a transitive, nonamenable, planar graph with one end. Let $\omega$ be an invariant percolation on $G$. Suppose that both $\omega$ and $\omega^{\dagger}$ have infinite components a.s. Then a.s. at least one among $\omega$ and $\omega^{\dagger}$ has infinitely many infinite components.

Proof. Draw $G$ and $G^{\dagger}$ in the plane in such a way that every edge $e$ intersects $e^{\dagger}$ in one point, $v_{e}$, and there are no other intersections of $G$ and $G^{\dagger}$. This defines a new graph $\widehat{G}$, whose vertices are $\mathrm{V}(G) \cup \mathrm{V}\left(G^{\dagger}\right) \cup\left\{v_{e}: e \in \mathrm{E}(G)\right\}$. Note that $\widehat{G}$ is quasi-transitive.

Set

$$
\widehat{\omega}:=\left\{\left[v, v_{e}\right] \in \mathrm{E}(\widehat{G}): v \in \mathrm{V}(G), e \in \omega\right\} \cup\left\{\left[v^{\dagger}, v_{e}\right] \in \mathrm{E}(\widehat{G}): v^{\dagger} \in \mathrm{V}\left(G^{\dagger}\right), e \notin \omega\right\} .
$$

Then $\widehat{\omega}$ is an invariant percolation on $\widehat{G}$. Note that the number of infinite components of $\widehat{\omega}$ is the number of infinite components of $\omega$ plus the number of infinite components of $\omega^{\dagger}$. By Lemma 3.5 applied to $\widehat{\omega}$, we find that $\widehat{\omega}$ has infinitely many infinite components.

Proof of Theorem 3.1. Each of $k, k^{\dagger}$ is in $\{0,1, \infty\}$ by Lemma 3.5. The case $\left(k, k^{\dagger}\right)=(0,0)$ is ruled out by Lemma 3.3. Since every two infinite components of $\omega$ must be separated by some component of $\omega^{\dagger}$, the situation $\left(k, k^{\dagger}\right)=(\infty, 0)$ is impossible. The same reasoning shows that $\left(k, k^{\dagger}\right)=(0, \infty)$ cannot happen. The case $\left(k, k^{\dagger}\right)=(1,1)$ is ruled out by Corollary 3.6 . 
Theorem 3.7. Let $G$ be a transitive, nonamenable, planar graph with one end, and let $\omega$ be Bernoulli $(p)$ bond percolation on $G$. Let $k$ be the number of infinite components of $\omega$, and let $k^{\dagger}$ be the number of infinite components of $\omega^{\dagger}$. Then a.s.

$$
\left(k, k^{\dagger}\right) \in\{(1,0),(0,1),(\infty, \infty)\} .
$$

Proof. By Theorem 3.1, it is enough to rule out the cases $(1, \infty)$ and $(\infty, 1)$. Let $K$ be a finite connected subgraph of $G$. If $K$ intersects two distinct infinite components of $\omega$, then $\omega^{\dagger}-\left\{e^{\dagger}: e \in \mathrm{E}(K)\right\}$ has more than one infinite component. If $k>1$ with positive probability, then there is some finite subgraph $K$ such that $K$ intersects two infinite components of $\omega$ with positive probability. Therefore, we find that $k^{\dagger}>1$ with positive probability (since the distribution of $\omega^{\dagger}-\left\{e^{\dagger}: e \in \mathrm{E}(K)\right\}$ is absolutely continuous to the distribution of $\left.\omega^{\dagger}\right)$. By ergodicity, this gives $k^{\dagger}>1$ a.s. An entirely dual argument shows that $k>1$ a.s. when $k^{\dagger}>1$ with positive probability.

Theorem 3.8. Let $G$ be a transitive, nonamenable, planar graph with one end. Then $p_{c}\left(G^{\dagger}\right)+p_{u}(G)=1$ for Bernoulli bond percolation.

Proof. Let $\omega_{p}$ be Bernoulli $(p)$ bond percolation on $G$. Then $\omega_{p}^{\dagger}$ is Bernoulli $(1-p)$ bond percolation on $G^{\dagger}$. It follows from Theorem 3.7 that the number of infinite components $k^{\dagger}$ of $\omega^{\dagger}$ is 1 when $p<p_{c}(G), \infty$ when $p \in\left(p_{c}(G), p_{u}(G)\right)$, and 0 when $p>p_{u}(G)$.

Proof of Theorem 1.1. We start with the proof for bond percolation. The easy inequality $p_{c}(G) \geq 1 /(d-1)$, where $d$ is the maximal degree of the vertices in $G$, is well known.

Set $p_{c}=p_{c}(G)$. By Theorem 1.3, $\omega_{p_{c}}$ has only finite components a.s. By Theorem 3.7, $\left(\omega_{p_{c}}\right)^{\dagger}$ has a unique infinite component a.s. Consequently, by Theorem 1.3 again, $\left(\omega_{p_{c}}\right)^{\dagger}$ is supercritical; that is, $p_{c}\left(G^{\dagger}\right)<1-p_{c}(G)$. An appeal to Theorem 3.8 now establishes the inequality $p_{c}(G)<p_{u}(G)$.

Since $p_{u}(G)=1-p_{c}\left(G^{\dagger}\right) \leq 1-1 /\left(d^{\dagger}-1\right)$, where $d^{\dagger}$ is the maximal degree of the vertices in $G^{\dagger}$, we get $p_{u}(G)<1$, and the proof for bond percolation is complete.

If $\omega$ is site percolation on $G$, let $\omega^{b}$ be the set of edges of $G$ with both endpoints in $\omega$. Then $\omega^{b}$ is a bond percolation on $G$. In this way, results for bond percolation can be adapted to site percolation. However, even if $\omega$ is Bernoulli, $\omega^{b}$ is not. Still, it is easy to check that the above proof applies also to $\omega^{b}$. The details are left to the reader.

Proof of Theorem 1.2. By Proposition 2.1, Aut $(G)$ is discrete and unimodular. By Theorem 3.8, $\left(\omega_{p_{u}}\right)^{\dagger}$ is critical Bernoulli bond percolation on $G^{\dagger}$. Hence, by Theorem 1.3, $\left(\omega_{p_{u}}\right)^{\dagger}$ has a.s. no infinite components. Therefore, it follows from Theorem 3.1 that $\omega_{p_{u}}$ has a single infinite component.

\section{Geometric consequences}

We now investigate briefly the geometry of percolation clusters on vertextransitive tilings of $\mathbb{H}^{2}$. Recall that the ideal boundary $\partial \mathbb{H}^{2}$ of $\mathbb{H}^{2}$ is homeomorphic to the circle $S^{1}$. Given a point $o \in \mathbb{H}^{2}, \partial \mathbb{H}^{2}$ can be identified with the space of infinite geodesic rays starting from $o$. Let $z_{n}$ be a sequence in $\mathbb{H}^{2}$. We say that $z_{n}$ converges to a point $z$ in $\partial \mathbb{H}^{2}$, if the geodesic segments $\left[o, z_{n}\right]$ converge to the ray corresponding to $z$. (That is, the length of $\left[o, z_{n}\right]$ tends to infinity and the angle at 
$o$ between the ray from $o$ corresponding to $z$ and the segment $\left[o, z_{n}\right]$ tends to zero.) One can show that the convergence of $z_{n}$ does not depend on the choice of $o$.

Theorem 4.1. Let $T$ be a vertex-transitive tiling of $\mathbb{H}^{2}$ with finite sided faces, let $G$ be the graph of $T$, and let $\omega$ be Bernoulli percolation on $G$. Almost surely, every infinite component of $\omega$ contains a path that has a unique limit point in the ideal boundary of $\mathbb{H}^{2}$.

In [BLS99] it is shown that for any unimodular transitive graph $G$ and every $p \in[0,1]$, a.s. every infinite component of $\operatorname{Bernoulli}(p)$ percolation on $G$ is transient and simple random walk on it has positive speed.

Proof. Suppose that $\omega$ has infinite components with positive probability. Let $X(t)$ be a simple random walk on an infinite component of $\omega$. Then, by the above result of [BLS99], a.s. there is a $\Lambda>0$ such that

$$
\operatorname{dist}(X(t), X(0)) \geq t \Lambda,
$$

for all sufficiently large $t$, where dist is the distance in the hyperbolic metric. Clearly, we also have

$$
\operatorname{dist}(X(t), X(s)) \leq L|t-s|,
$$

for some constant $L$. These inequalities are enough to conclude that $X(t)$ tends to a limit in the ideal boundary. Indeed, fix a polar coordinate system $(r, \theta)$, where $r=r(p)$ is the hyperbolic distance $\operatorname{dist}(X(0), p)$ from $p$ to $X(0)$ and $\theta$ is the angle between the segment $[X(0), p$,$] and some fixed ray starting at X(0)$. Note that there are constants $c<1<C$ such that for points $p, q \in \mathbb{H}^{2}$

$$
d(\theta(p), \theta(q)) \leq C c^{r(p)-\operatorname{dist}(p, q)}
$$

where the distance $d(\theta(p), \theta(q))$ refers to the arclength distance on the unit circle. It therefore follows immediately from (4.1) and (4.2) that $\{\theta(X(t))\}$ is a Cauchy sequence; that is, $\lim _{t} \theta(X(t))$ exists. This implies that $X(t)$ tends to a limit in the ideal boundary.

There is also a proof that does not use speed, but instead uses the easier result from [BLS99] that the infinite components are transient. We now give that proof.

Recall that a metric $d_{0}$ on the vertices of a graph $H$ is proper, if every ball of finite radius contains finitely many vertices.

Lemma 4.2. Let $G$ be an infinite connected (locally finite) graph. Then $G$ is transient iff every proper metric on $G$ satisfies

$$
\sum_{[v, u] \in \mathrm{E}(G)} d_{0}(v, u)^{2}=\infty .
$$

This easy observation is certainly not new.

Proof. A function $f: \vee(G) \rightarrow \mathbb{R}$ is proper if $f^{-1}(K)$ is finite for every compact $K$. The Dirichlet energy of $f$ is $\mathcal{D}(f):=\sum_{[v, u] \in \mathrm{E}(G)}(f(u)-f(v))^{2}$. It is well known that $G$ is recurrent iff there is a proper function $f: \mathrm{V}(G) \rightarrow[0, \infty)$ with finite Dirichlet energy. (See [DS84] or [Lyo01].)

Suppose that $f$ is proper and has finite Dirichlet energy. Without loss of generality, we may assume that $f(v) \neq f(u)$ if $v \neq u$. Then define $d_{0}(v, u):=|f(v)-f(u)|$. In the other direction, given a proper metric $d_{0}$, set $f(u)=d_{0}(o, u)$, where $o \in \mathrm{V}(G)$ is arbitrary. By the triangle inequality it follows that $\mathcal{D}(f)<\infty$. 
Second proof of Theorem 4.1. We first prove that every transient connected subgraph $H \subset G$ has a path with a limit in the ideal boundary. Indeed, consider the Poincaré (disk) model for the hyperbolic plane $\mathbb{H}^{2}$. Given two points, $x, y \in \mathbb{H}^{2}$, let $d_{E}(x, y)$ be the Euclidean distance from $x$ to $y$. Let $d_{E}^{H}$ be the maximal metric on $\mathrm{V}(H)$ such that $d_{E}^{H}(v, u) \leq d_{E}(v, u)$ whenever $[v, u] \in \mathrm{E}(H)$ (in other words, $d_{E}^{H}(v, u)=\inf \sum_{j=1}^{n} d_{E}\left(v_{j-1}, v_{j}\right)$, where the infimum is taken with respect to all finite paths $v_{0}, v_{1}, \ldots, v_{n}$ in $H$ from $v$ to $u$ ).

We now show that

$$
\sum_{[v, u] \in \mathrm{E}(G)} d_{E}(v, u)^{2}<\infty
$$

Let $o \in \mathrm{V}(G)$ be a basepoint, and for every neighbor $u$ of $o$ let $D_{u}$ be a compact disk in $\mathbb{H}^{2}$ whose boundary contains the points $o$ and $u$. Let $P$ be the collection of all disks of the form $\gamma D_{u}$ where $u$ neighbors with $o$ and $\gamma \in \Gamma$ is an automorphism of $G$ acting on $\mathbb{H}^{2}$ as an isometry. Since $\Gamma$ acts discretely on $\mathbb{H}^{2}$, there is a finite upper bound $M$ for the number of disks in $P$ that contain any point $z \in \mathbb{H}^{2}$. Since each disk in $P$ is contained in $\mathbb{H}^{2}$, it follows that the sum of the Euclidean areas of the disks in $P$ is finite. As the square of the Euclidean diameter of a disk is linearly related to the Euclidean area, this proves (4.3).

Because $H$ is transient, Lemma 4.2 implies that the metric $d_{E}^{H}$ is not proper. Hence there is an infinite simple path in $H$ with finite $d_{E}$-length. Theorem 4.1 follows, because a.s. the infinite components of $\omega$ are transient, by [BLS99].

Lemma 4.3. Let $T$ be a vertex-transitive tiling of $\mathbb{H}^{2}$ with finite sided faces, let $G$ be the graph of $T$, and let $\omega$ be an invariant percolation on $G$. Let $Z$ be the set of points $z$ in the ideal boundary $\partial \mathbb{H}^{2}$ such that there is a path in $\omega$ with limit $z$. Then a.s. $Z=\varnothing$ or $Z$ is dense in $\partial \mathbb{H}^{2}$.

Proof. Given a vertex $v \in \mathrm{V}(G)$, we may consider a polar coordinate system with $v$ as the origin, and with respect to this coordinate system $\partial \mathbb{H}^{2}$ can be thought of as a metric circle of circumference $2 \pi$. Let $d_{v}$ denote this metric of $\partial \mathbb{H}^{2}$, and let $a(v)$ be the length of the largest component of $\partial \mathbb{H}^{2} \backslash Z$, with respect to $d_{v}$. Note that for vertices $v \in \mathrm{V}(G)$, the law of the random variable $a(v)$ does not depend on $v$. Let $o \in \mathrm{V}(G)$, let $\epsilon \in(0,1)$, and let $\delta$ be the probability that $\epsilon<a(o)<2 \pi-\epsilon$.

Suppose that $\delta>0$. Let $R>0$ be very large, and let $x$ be a random-uniform point on the circle of radius $R$ about $o$ in $\mathbb{H}^{2}$. Let $v_{x}$ be the vertex of $G$ closest to $x$. On the event $\epsilon<a(o)<2 \pi-\epsilon$, there is probability greater than $\epsilon /(4 \pi)$ that the geodesic ray from $o$ containing $x$ hits $\partial \mathbb{H}^{2}$ at a point $x^{\prime}$ with $d_{o}\left(x^{\prime}, Z\right) \geq \epsilon / 4$; this happens when $x^{\prime}$ is within the inner half of the largest arc of $\partial \mathbb{H}^{2} \backslash Z$ with respect to $d_{o}$. On that event, if $R$ is very large (as a function of $\epsilon$ ), we have $a\left(v_{x}\right)$ as close as we wish to $2 \pi$, and $a\left(v_{x}\right) \neq 2 \pi$. However, since

$$
\mathbf{P}\left[a\left(v_{x}\right) \in(2 \pi-t, 2 \pi)\right]=\mathbf{P}[a(o) \in(2 \pi-t, 2 \pi)] \rightarrow 0, \quad \text { as } t \searrow 0,
$$

it follows that $\mathbf{P}[a(o) \in(\epsilon, 2 \pi-\epsilon)]=0$. Consequently, $a(o) \in\{0,2 \pi\}$ a.s.

It remains to deal with the case that $Z$ is a single point with positive probability. But this is impossible, since the law of this point would be a finite positive measure on $\partial \mathbb{H}^{2}$ which is invariant under the automorphisms of $T$, and such a measure does not exist. (To verify this, observe that such a measure can have no atoms, since the orbit of any point in $\partial \mathbb{H}^{2}$ is infinite. Given any finite positive atomless measure on $\mathbb{H}^{2}$ and a small $\epsilon>0$, there is a bounded set of points $x \in \mathbb{H}^{2}$ with the property that 
for every half-plane which contains $x$, the arc of $\partial \mathbb{H}^{2}$ associated with it has measure at least $\epsilon$. However, the orbit of every point $x \in \mathbb{H}^{2}$ under the automorphisms of $T$ is infinite.)

Corollary 4.4. Let $T$ be a vertex-transitive tiling of $\mathbb{H}^{2}$ with finite sided faces, let $G$ be the graph of $T$, and let $\omega$ be Bernoulli percolation on $G$. If $\omega$ has infinite components a.s., then for every half-space $W \subset \mathbb{H}^{2}$, a.s. $\omega \cap W$ has infinite components.

The significance of percolation in hyperbolic half-spaces was noted by [Lal98].

Proof. This follows immediately from Theorem 4.1 and Lemma 4.3.

Corollary 4.5 (Connectivity decay). Let $T$ be a vertex-transitive tiling of $\mathbb{H}^{2}$ with finite sided faces, let $G$ be the graph of $T$, let $p<p_{u}(G)$, and let $\omega=\omega_{p}$ be Bernoulli $(p)$ percolation on $G$. Let $\tau(v, u)$ denote the probability that $v$ and $u$ are in the same cluster of $\omega$. There is some $a=a(G, p)<1$ such that $\tau(v, u) \leq a^{d(v, u)}$ for every $v, u \in \mathrm{V}(G)$.

Proof. There are constants $b=b(G, p)$ and $c=c(G, p)>0$ such that if $L$ and $L^{\prime}$ are two hyperbolic lines and the minimal distance between $L$ and $L^{\prime}$ is at least $b$, then with probability at least $c$ there is an infinite path in $\omega^{\dagger}$ which separates $L$ from $L^{\prime}$ in $\mathbb{H}^{2}$ and does not intersect $L \cup L^{\prime}$. This follows from Corollary 4.4 (or rather its generalization to the quasi-transitive setting), since, when $b$ is large enough, for given $L$ and $L^{\prime}$, there are hyperbolic half-spaces $H$ and $H^{\prime}$, disjoint from $L \cup L^{\prime}$, such that $H \cup H^{\prime}$ separates $L$ from $L^{\prime}$. Moreover, the automorphisms of $G$ act co-compactly on $\mathbb{H}^{2}$, so it is enough to consider a compact collection of pairs $\left(L, L^{\prime}\right)$.

If $v, u \in \mathrm{V}(G)$ and $d(v, u)$ is large, then one can find a collection of hyperbolic lines $L_{0}, L_{1}, \ldots, L_{m}$, with $d(v, u) \leq O(1) m$, such that the distance between $L_{j}$ and $L_{j+1}$ is $b$, and for each $j=1,2, \ldots, m$, the line $L_{j}$ separates $\{v\} \cup L_{0} \cup \cdots \cup L_{j-1}$ from $L_{j+1} \cup \cdots \cup L_{m} \cup\{u\}$. The corollary immediately follows, since, by independence, with probability at least $1-(1-c)^{m}$ there is some $j=1,2, \ldots, m$ and a path $\gamma \subset \omega^{\dagger}$ which separates $L_{j-1}$ from $L_{j}$, and thereby separates $u$ from $v$.

\section{Mass transport in the hyperbolic Plane And some APplications}

The Mass Transport Principle [Häg97] has an important role in the study of percolation on nonamenable transitive graphs. See, e.g., [BLPS99a]. We now develop a continuous version of this principle, in the setting of the hyperbolic plane, and later produce several applications.

Definition 5.1. A diagonally-invariant measure $\mu$ on $\mathbb{H}^{2} \times \mathbb{H}^{2}$ is a measure satisfying

$$
\mu(g A \times g B)=\mu(A \times B)
$$

for all measurable $A, B \subset \mathbb{H}^{2}$ and $g \in \operatorname{Isom}\left(\mathbb{H}^{2}\right)$.

Theorem 5.2 (Mass Transport Principle in $\mathbb{H}^{2}$ ). Let $\mu$ be a nonnegative diagonally-invariant Borel measure on $\mathbb{H}^{2} \times \mathbb{H}^{2}$. Suppose that $\mu\left(A \times \mathbb{H}^{2}\right)<\infty$ for some open $A \subset \mathbb{H}^{2}$. Then

$$
\mu\left(B \times \mathbb{H}^{2}\right)=\mu\left(\mathbb{H}^{2} \times B\right)
$$


for all measurable $B \subset \mathbb{H}^{2}$. Moreover, there is a constant $c$ such that $\mu\left(B \times \mathbb{H}^{2}\right)=$ $c$ area $(B)$ for all measurable $B \subset \mathbb{H}^{2}$.

The same conclusions apply in the case where $\mu$ is a diagonally-invariant Borel signed measure on $\mathbb{H}^{2} \times \mathbb{H}^{2}$, provided that $|\mu|\left(K \times \mathbb{H}^{2}\right)<\infty$ for every compact $K \subset \mathbb{H}^{2}$.

Recall that the subgroup $\operatorname{Isom}_{+}\left(\mathbb{H}^{2}\right)$ of orientation preserving isometries is a simple group. Consequently, $\operatorname{Isom}_{+}\left(\mathbb{H}^{2}\right)$ must be contained in the kernel of the modular function, which is a homomorphism from $\operatorname{Isom}\left(\mathbb{H}^{2}\right)$ to the multiplicative group $\mathbb{R}_{+}$. It follows that the modular function is identically 1 ; that is, $\operatorname{Isom}\left(\mathbb{H}^{2}\right)$ is unimodular. Let $\eta$ denote Haar measure of $\operatorname{Isom}\left(\mathbb{H}^{2}\right)$. The particular consequence of unimodularity that we shall need is that $\eta(A)=\eta\left\{g^{-1}: g \in A\right\}$ for every measurable $A \subset \operatorname{Isom}\left(\mathbb{H}^{2}\right)$. (Indeed, set $\nu(A):=\eta\left\{g^{-1}: g \in A\right\}$. Then it is easy to see that $\nu$ is left-invariant, and by the uniqueness of Haar measure it must be a multiple of $\eta$. By choosing $A$ symmetric with respect to $g \rightarrow g^{-1}$, it follows that $\nu=\eta$.)

Proof. We first prove the nonnegative case. Note that $\nu(B)=\mu\left(B \times \mathbb{H}^{2}\right)$ is an Isom $\left(\mathbb{H}^{2}\right)$-invariant Borel measure on $\mathbb{H}^{2}$. Hence $\nu=c$ area, for some constant c. Suppose for the moment that $\mu\left(\mathbb{H}^{2} \times B\right)$ is finite for some open $B$. Then $\mu\left(\mathbb{H}^{2} \times B\right)=c^{\prime}$ area $(B)$, and it remains to show that $c=c^{\prime}$.

Let $B$ be some open ball in $\mathbb{H}^{2}$. Fix a point $o \in \mathbb{H}^{2}$, and let $\eta$ be Haar measure on $\operatorname{Isom}\left(\mathbb{H}^{2}\right)$. Note that $\eta\{g: z \in g B\}=\eta\{g: o \in g B\}$ when $z \in \mathbb{H}^{2}$. Hence, Fubini gives

$$
\int \mu((g B) \times B) d \eta(g)=\int_{(z, g)} 1_{z \in g B} d \mu(\{z\} \times B) d \eta(g)=\mu\left(\mathbb{H}^{2} \times B\right) \eta\{g: o \in g B\} .
$$

Therefore,

$$
\mu\left(\mathbb{H}^{2} \times B\right)=\frac{\int \mu((g B) \times B) d \eta(g)}{\eta\{g: o \in g B\}}=\frac{\int \mu\left(B \times\left(g^{-1} B\right)\right) d \eta(g)}{\eta\{g: o \in g B\}}=\mu\left(B \times \mathbb{H}^{2}\right) .
$$

To complete the proof for the nonnegative case, we only need to show that $\mu\left(\mathbb{H}^{2} \times B\right)<\infty$ for some open $B$. Indeed, for every $r>0$ let $F_{r}$ be the set of $(x, y) \in \mathbb{H}^{2} \times \mathbb{H}^{2}$ such that $d(x, y)<r$. Set $\mu_{r}(K)=\mu\left(K \cap F_{r}\right)$. Then $\mu_{r}$ is diagonally invariant and the above proof applies to it. Therefore,

$$
\mu\left(\mathbb{H}^{2} \times B\right)=\sup _{r>0} \mu_{r}\left(\mathbb{H}^{2} \times B\right)=\sup _{r>0} \mu_{r}\left(B \times \mathbb{H}^{2}\right)=\mu\left(B \times \mathbb{H}^{2}\right) .
$$

This completes the proof in the nonnegative case, and the signed version easily follows by decomposing the measure as a difference of two nonnegative measures.

Of course, there is nothing special about the hyperbolic plane in this case; there is a similar version of the Mass Transport Principle in any symmetric space.

Also, the Mass Transport Principle holds when the assumption that $\mu$ is invariant under the diagonal action of $\operatorname{Isom}\left(\mathbb{H}^{2}\right)$ is replaced by the weaker assumption that $\mu$ is invariant under the diagonal action of $\operatorname{Isom}_{+}\left(\mathbb{H}^{2}\right)$, the group of orientation preserving isometries. Similarly, the results stated below assuming invariance under $\operatorname{Isom}\left(\mathbb{H}^{2}\right)$ are equally valid assuming $\operatorname{Isom}_{+}\left(\mathbb{H}^{2}\right)$-invariance.

We now illustrate the Mass Transport Principle with an application involving a relation between the densities of vertices, edges, and faces in tilings. 
Let $T$ be a random tiling of $\mathbb{H}^{2}$, whose law is invariant under Isom $\left(\mathbb{H}^{2}\right)$, and with the property that a.s. the edges in $T$ are piecewise smooth, and each face and each vertex has a finite number of edges incident with it.

Definition 5.3 (Vertex, face, and edge densities). How does one measure the abundance of vertices in $T$ ? Given a measurable set $A \subset \mathbb{H}^{2}$, let $N_{v}(A)$ be the number of vertices of $T$ in $A$. If $\mathbf{E} N_{v}(A)$ is finite for every measurable bounded $A \subset \mathbb{H}^{2}$, then we say that $T$ has finite vertex density. In this case, $\mu(A):=\mathbf{E} N_{v}(A)$ is clearly an invariant Borel measure; hence there is a constant $D_{\vee}$ such that $\mathbf{E} N_{v}=D_{\vee}$ area. This number $D_{\vee}$ will be called the vertex density of $T$. The definitions of the face and edge densities are a bit more indirect. Fix a point $o \in \mathbb{H}^{2}$. The face density is defined by $D_{\mathrm{F}}=\mathbf{E}\left[A_{o}^{-1}\right]$, where $A_{o}$ is the area of the tile of $T$ that contains $o$. Given a measurable set $A \subset \mathbb{H}^{2}$, let $Y_{e}(A)$ be the sum of the degrees of the vertices of $T$ in $A$. If $\mathbf{E} Y_{e}(A)$ is finite for bounded measurable $A$, and $\mathbf{E} Y_{e}=2 D_{\mathrm{E}}$ area, then we say that $T$ has finite edge density $D_{\mathrm{E}}$. It is left to the reader to convince herself or himself that these definitions are reasonable.

Given a set $B \subset \mathbb{H}^{2}$ with reasonably smooth boundary (piecewise $C^{2}$ is enough), let $\kappa_{\partial B}$ denote the associated curvature measure. That is, for all open $A \subset \mathbb{H}^{2}$, $\kappa_{\partial B}(A)$ is the (signed) curvature of $A \cap \partial B$. Note that if $p$ is a point on $\partial B$ and the internal angle of $B$ at $p$ is $\alpha$, then $\kappa_{\partial B}(\{p\})=\pi-\alpha$. The following instance of the Gauss Bonnet Theorem will be very useful for us.

Theorem 5.4 (Gauss-Bonnet in $\mathbb{H}^{2}$ ). Let $A$ be a closed topological disk in $\mathbb{H}^{2}$, whose boundary is a piecewise smooth simple closed path. Then

$$
2 \pi+\operatorname{area}(A)=\kappa_{\partial A}(\partial A) .
$$

Suppose that $K \subset \mathbb{H}^{2}$ is a compact set whose boundary $\partial K$ is a 1-manifold. If $\partial K$ has finite unsigned curvature, namely $\left|\kappa_{\partial K}\right|\left(\mathbb{H}^{2}\right)<\infty$, then set

$$
\mu_{K}(A \times B):=\frac{\operatorname{area}(A \cap K)}{\operatorname{area}(K)} \kappa_{\partial K}(B),
$$

for every measurable $A, B \subset \mathbb{H}^{2}$. This is a signed measure on $\mathbb{H}^{2} \times \mathbb{H}^{2}$, and will be very useful below. Note that $\mu_{K}\left(\mathbb{H}^{2} \times B\right)=\kappa_{\partial K}(B)$.

Given a tiling $T$ of $\mathbb{H}^{2}$, let $\mathrm{F}(T)$ denote the set of faces (that is, tiles) of $T$, and set

$$
\mu_{T}=\sum_{f \in \mathrm{F}(T)} \mu_{f}, \quad \widehat{\mu}_{T}=\sum_{f \in \mathrm{F}(T)}\left|\mu_{f}\right| .
$$

If $T$ is a random tiling such that

$$
\mathbf{E} \widehat{\mu}_{T}\left(\mathbb{H}^{2} \times A\right)<\infty
$$

for bounded open sets $A$, then we say that $T$ has locally integrable curvature.

Theorem 5.5 (Euler formula for random tilings in $\mathbb{H}^{2}$ ). Let $T$ be a random tiling of $\mathbb{H}^{2}$, whose distribution is $\operatorname{Isom}\left(\mathbb{H}^{2}\right)$-invariant. Suppose that a.s. each face of $T$ is a closed topological disk with piecewise smooth boundary, and each vertex has degree at least 3. Further suppose that $T$ has locally integrable curvature. Then $T$ has finite vertex, edge, and face densities, and these densities satisfy the relation

$$
2 \pi\left(D_{\mathrm{F}}-D_{\mathrm{E}}+D_{\mathrm{V}}\right)=-1 .
$$


Proof. Suppose that $\gamma$ is an arc on the boundary of two tiles, $f$ and $f^{\prime}$, of $T$, and that $\gamma$ is disjoint from the vertices of $T$. Then $\kappa_{f}(\gamma)=-\kappa_{f^{\prime}}(\gamma)$, as the curvature negates under a change of orientation. Consequently, if $A \subset \mathbb{H}^{2}$ is disjoint from the vertices of $T$, then $\mu_{T}\left(\mathbb{H}^{2} \times A\right)=0$. On the other hand, if $v$ is a vertex of a face $f$, then $\mu_{f}\left(\mathbb{H}^{2} \times\{v\}\right)$ is equal to $\pi$ minus the interior angle of $f$ at $v$. Consequently, $\mu_{T}\left(\mathbb{H}^{2} \times\{v\}\right)=\pi\left(\operatorname{deg}_{v}-2\right)$. Since $T$ has locally integrable curvature and every vertex has degree at least 3 , this shows that $T$ has finite vertex and edge density, and that

$$
\mathbf{E} \mu_{T}\left(\mathbb{H}^{2} \times A\right)=2 \pi\left(D_{\mathrm{E}}-D_{\mathrm{V}}\right) \operatorname{area}(A) .
$$

By the Mass Transport Principle,

$$
\mathbf{E} \mu_{T}\left(A \times \mathbb{H}^{2}\right)=2 \pi\left(D_{\mathrm{E}}-D_{\mathrm{V}}\right) \operatorname{area}(A) .
$$

On the other hand, Gauss-Bonnet shows that when $A$ is contained in a face $f \in$ $\mathrm{F}(T)$, we have $\mu_{T}\left(A \times \mathbb{H}^{2}\right)=\operatorname{area}(A)(2 \pi+\operatorname{area}(f)) /$ area $(f)$. Consequently,

$$
\operatorname{area}(A)\left(2 \pi D_{\mathrm{F}}+1\right)=\mathbf{E} \mu_{T}\left(A \times \mathbb{H}^{2}\right)=2 \pi\left(D_{\mathrm{E}}-D_{\mathrm{V}}\right) \operatorname{area}(A) .
$$

Remark 5.6. As the proof shows, the -1 on the right side of (5.2) comes from the curvature of $\mathbb{H}^{2}$. An analogous equation holds in $\mathbb{R}^{2}$ and in $S^{2}$, with the -1 changed to 0 and 1, respectively. (For $S^{2}$ this is just the classical Euler formula.) A similar proof applies to $S^{2}$ and $\mathbb{R}^{2}$, but for these spaces one can also replace the use of the Mass Transport Principle with amenability.

Remark 5.7. When a.s. all the vertices in $T$ have degree 3 , we have $2 D_{\mathrm{E}}=3 D_{\mathrm{V}}$, and therefore (5.2) simplifies to

$$
D_{\vee}=2 D_{\mathrm{F}}+\frac{1}{\pi}
$$

\section{Voronol PERCOLATION IN THE HyPERBolic PLANE}

We now describe a very natural continuous percolation process in the hyperbolic plane.

Given a discrete nonempty set of points $X \subset \mathbb{H}^{2}$, the associated Voronoi tiling of $\mathbb{H}^{2}$ is defined by $T=T(X)=\left\{T_{x}: x \in X\right\}$, where

$$
T_{x}:=\left\{y \in \mathbb{H}^{2}: \operatorname{dist}(y, x)=\operatorname{dist}(y, X)\right\} .
$$

The point $x \in X$ is called the nucleus of $T_{x}$. Fix some parameters $p \in[0,1]$ and $\lambda \geq 0$. Let $W$ be a Poisson point process in $\mathbb{H}^{2}$ with intensity $\lambda_{W}:=p \lambda$, and let $B$ be an independent Poisson point process with intensity $\lambda_{B}:=(1-p) \lambda$. Note that $X:=W \cup B$ is a Poisson point process with intensity $\lambda$, and, given $X$, each point $x \in X$ is in $W$ with probability $p$, independently. Let $T=T(X)$ be the Voronoi tiling associated with $X$, and set

$$
\widehat{W}:=\bigcup_{w \in W} T_{w}, \quad \widehat{B}:=\bigcup_{b \in B} T_{b} .
$$

Observe that a.s. each vertex of the tiling $T(X)$ has degree 3. A.s. $\mathbb{H}^{2}$ is the union of $\widehat{W}$ and $\widehat{B}$, and $\widehat{W} \cap \widehat{B}$ is a 1-manifold. This model will be referred to as $(p, \lambda)$ Poisson-Voronoi-Bernoulli percolation in $\mathbb{H}^{2}$, or just Voronoi percolation, for short. The connected components of $\widehat{W}$ and of $\widehat{B}$ will be called clusters. 
It is clear that if $\widehat{W}$ has infinite components with positive probability, then it has infinite components a.s. Set

$$
\begin{aligned}
\mathcal{W} & :=\{(p, \lambda) \in[0,1] \times(0, \infty): \widehat{W} \text { has infinite components a.s. }\} \\
\mathcal{B} & :=\{(p, \lambda) \in[0,1] \times(0, \infty): \widehat{B} \text { has infinite components a.s. }\} .
\end{aligned}
$$

For every $\lambda>0$ define

$$
p_{c}(\lambda):=\inf \{p \in[0,1]:(p, \lambda) \in \mathcal{W}\} .
$$

It is clear that $(p, \lambda) \in \mathcal{W}$ if $p>p_{c}(\lambda)$.

Proof of Theorem 1.4. It is easy to adapt the proof of Theorem 3.7 to this setting. The details are left to the reader.

As we shall see, $[0,1] \times(0, \infty)=\mathcal{W} \cup \mathcal{B}$, so Theorem 1.4 covers all possibilities.

It is easy to see that in $\mathcal{W} \cap \mathcal{B}$ a.s. all unbounded components of $\widehat{W}$ have a cantor set of limit points in $\partial \mathbb{H}^{2}$, with dimension smaller than 1 .

Remark 6.1. One can easily show that the face density $D_{\mathrm{F}}$ of $T$ is $\lambda$, using the following mass transport. For each tile $f$ of $T$ with nucleus $x$ let $\nu_{f}\left(A \times A^{\prime}\right)=$ $\operatorname{area}(A \cap f) / \operatorname{area}(f)$ if $x \in A^{\prime}$ and 0 otherwise. Set $\nu_{T}=\sum_{f} \nu_{f}$. Then the Mass Transport Principle with $\mathbf{E} \nu_{T}$ gives $\lambda=D_{\mathrm{F}}$.

Theorem 6.2. $(1 / 2, \lambda) \in \mathcal{W} \cap \mathcal{B}$ for every $\lambda \in(0, \infty)$.

We present two proofs of this theorem, one uses the Mass Transport Principle, and the other uses hyperbolic surfaces. We start with the latter.

Hyperbolic Surfaces Proof. Fix some large $d>0$. Let $S$ be a compact hyperbolic surface, whose injectivity radius is greater than $5 d$; that is, any disk of radius $5 d$ in $S$ is isometric to a disk in the hyperbolic plane. It is well known that such surfaces exist. (See, e.g., Proposition 1 and Lemma 2 from [SS97].)

Consider $(1 / 2, \lambda)$ percolation in $S$. Let $K$ be the union of all white or black clusters of diameter less than $d$. We claim that each component of $K$ has diameter less than $d$. Indeed, if $A$ is a white or black cluster with diameter less than $d$, then $A$ is contained in a disk in $S$ which is isometric to a disk in the hyperbolic plane. It follows that the complement of $A$ consists of one component of diameter greater than $d$, and possibly several components of diameter smaller than $d$. So, if a black and a white cluster have diameters $<d$ and are adjacent, then one of them 'surrounds' the other, in the sense that the latter is contained in a component of the complement of the first which has diameter $<d$. It follows that each component of $K$ indeed has diameter $<d$.

For all $t>0$ let $K_{t}$ be the set of points in $K$ with distance at least $t$ to $S-K$. Because each component of $K$ is isometric to a set in the hyperbolic plane, the linear isoperimetric inequality for the hyperbolic plane implies that

$$
\text { length }\left(\partial K_{\delta}\right) \geq c \text { area } K_{\delta}
$$

holds for all $\delta \geq 0$, where $c>0$ is some fixed constant. Consequently,

$$
\begin{aligned}
\operatorname{area}(S)-\operatorname{area}\left(K_{1}\right) & \geq \operatorname{area}\left(K-K_{1}\right)=\int_{0}^{1}\left|\frac{d}{d t} \operatorname{area}\left(K_{t}\right)\right| d t \\
& =\int_{0}^{1} \operatorname{length} \partial K_{t} d t \geq c \int_{0}^{1} \operatorname{area}\left(K_{t}\right) d t \geq c \operatorname{area}\left(K_{1}\right),
\end{aligned}
$$


which implies

$$
\operatorname{area}\left(K_{1}\right) \leq(1+c)^{-1} \operatorname{area}(S) .
$$

Therefore, a uniform-random point in $S$ has probability at least $1-(1+c)^{-1}$ to be within distance 1 of a cluster with diameter $\geq d$. Because the injectivity radius of $S$ is $\geq 5 d$, the same would be true for an arbitrary point in the hyperbolic plane. Letting $d \rightarrow \infty$, we see that for any fixed point in $\mathbb{H}^{2}$, the probability that it is within distance 1 of an unbounded cluster is at least $1-(1+c)^{-1}$. This implies that $(1 / 2, \lambda) \in \mathcal{W} \cup \mathcal{B}$, and hence $(1 / 2, \lambda) \in \mathcal{W} \cap \mathcal{B}$, by symmetry.

Mass Transport Proof. Consider Voronoi percolation with parameters $(1 / 2, \lambda)$. Let $d>0$ be large, and let $F_{d}$ be the union of all black or white components of diameter less than $d$. Then each component $K$ of $F_{d}$ is a.s. a topological disk with diameter bounded by $d$.

Given a component $K$ of $F_{d}$, as above, let $\mu_{K}$ be the signed measure on $\mathbb{H}^{2} \times \mathbb{H}^{2}$ defined by

$$
\mu_{K}(A \times B)=\frac{\operatorname{area}(A \cap K)}{\operatorname{area}(K)} \kappa_{\partial K}(B),
$$

where $\kappa_{\partial K}$ is the curvature measure on $\partial K$. Given the percolation configuration $X$, let

$$
\mu^{X}:=\sum_{K} \mu_{K}, \quad \mu:=\mathbf{E} \mu^{X},
$$

where the sum extends over all components $K$ of $F_{d}$. Note that $\left|\mu^{X}\left(\mathbb{H}^{2} \times A\right)\right|$ is bounded by $2 \pi$ times the number of vertices of the Voronoi tiling that are in the intersection of $A$ with the boundary of $F_{d}$. Consequently, $|\mu|\left(\mathbb{H}^{2} \times A\right)$ is finite for every bounded $A$. The measure $\mu$ is clearly invariant under the diagonal action of Isom $\left(\mathbb{H}^{2}\right)$ on $\mathbb{H}^{2} \times \mathbb{H}^{2}$. Therefore, the Mass Transport Principle applies to $\mu$.

Fix some point $o \in \mathbb{H}^{2}$. Fubini and the Gauss Bonnet Theorem 5.4 show that $\mu\left(A \times \mathbb{H}^{2}\right) \geq \operatorname{area}(A) \mathbf{P}\left[o \in F_{d}\right]$. By the Mass Transport Principle, we therefore have

$$
\mu\left(\mathbb{H}^{2} \times A\right) \geq \operatorname{area}(A) \mathbf{P}\left[o \in F_{d}\right] .
$$

Fix some $A$ with area $(A)>0$. Let $F_{\infty}=\bigcup_{d>0} F_{d}$. From (6.1) we find that

$2 \pi \mathbf{E} \mid\left\{\right.$ Voronoi vertices in $\left.A \cap \partial F_{d}\right\} \mid \geq \operatorname{area}(A) \mathbf{P}\left[o \in F_{d}\right]$.

Because $\mathbf{E} \mid\{$ Voronoi vertices in $A\} \mid<\infty$, by letting $d \rightarrow \infty$ it follows that

$$
2 \pi \mathbf{E} \mid\left\{\text { Voronoi vertices in } A \cap \partial F_{\infty}\right\} \mid \geq \operatorname{area}(A) \mathbf{P}\left[o \in F_{\infty}\right] .
$$

This shows that $\partial F_{\infty}$ is not empty with positive probability. On this event, $\widehat{W}$ has an unbounded component or $\widehat{B}$ has an unbounded component. This gives $(1 / 2, \lambda) \in \mathcal{W} \cup \mathcal{B}$. Symmetry then implies that $(1 / 2, \lambda) \in \mathcal{W} \cap \mathcal{B}$, which completes the proof.

Note that the latter proof does not require that the tile colors be independent. In fact, it gives the following generalization.

Theorem 6.3. Suppose that $Z \subset \mathbb{H}^{2}$ is a closed random subset whose distribution is $\operatorname{Isom}\left(\mathbb{H}^{2}\right)$-invariant, such that $\partial Z$ is a.s. a 1-manifold and $\mathbf{E}\left[\left|\kappa_{\partial Z}\right|(A)\right]<\infty$ for some nonempty open $A \subset \mathbb{H}^{2}$. Then a.s. there is an infinite component in $Z$ or in $\mathbb{H}^{2} \backslash Z$. 
We now work a bit harder to get the following explicit upper bound on $p_{c}(\lambda)$. The bound will also show that $p_{c}(\lambda) \rightarrow 0$ as $\lambda \searrow 0$.

\section{Theorem 6.4.}

$$
\forall \lambda>0, \quad p_{c}(\lambda) \leq \frac{1}{2}-\frac{1}{4 \lambda \pi+2} .
$$

Note that the right hand side is zero at $\lambda=0$ and its derivative there is $\pi$.

Proof. Let $\lambda>0$, and let $p<p_{c}(\lambda)$. Then $p<1 / 2$, by Theorem 6.2. Consider three independent Poisson point processes $W, B, R$ in $\mathbb{H}^{2}$, with (positive) intensities $p \lambda, p \lambda$, and $(1-2 p) \lambda$, respectively. Then the intensity of $W \cup B \cup R$ is $\lambda$. Let $X=(W, B, R)$, and consider the Voronoi tiling $T=T(W \cup B \cup R)$. Color the tiles with white, black, or red, depending on whether the nucleus is in $W, B$, or $R$, respectively. Let

$$
p_{R}:=1-2 p, \quad p_{W}:=p, \quad p_{B}:=p .
$$

Then, given $W \cup B \cup R, p_{r}, p_{W}$, and $p_{B}$ are the probabilities that any given tile of $T(W \cup B \cup R)$ is red, white, and black, respectively.

Because $p<p_{c}$, a.s. there are no unbounded white or black clusters. Given a cluster $K$, let the hull of $K$ be the union of $K$ with the bounded components of $\mathbb{H}^{2}-K$. Call a white or black cluster $K$ an empire if there is no black or white cluster $K^{\prime}$ such that the hull of $K^{\prime}$ contains $K$. Let $\mathcal{K}$ be the set of all hulls of empires.

Condition on the triplet $X=(W, B, R)$, and let $K \in \mathcal{K}$. Let $\kappa_{\partial K}$ denote the curvature measure on $\partial K$. Let $\mu_{K}$ be the signed measure on $\mathbb{H}^{2} \times \mathbb{H}^{2}$ defined by

and let

$$
\mu_{K}\left(A \times A^{\prime}\right)=\frac{\operatorname{area}(A \cap K)}{\operatorname{area}(K)} \kappa_{\partial K}\left(A^{\prime}\right),
$$

$$
\mu^{X}=\sum_{K \in \mathcal{K}} \mu_{K}, \quad \mu=\mathbf{E} \mu^{X},
$$

as above. As before, it is not hard to verify that $|\mu|\left(\mathbb{H}^{2} \times A\right)$ is finite for every bounded measurable $A$.

Fix a point $o \in \mathbb{H}^{2}$. The Gauss Bonnet Theorem 5.4 shows that

$$
\mu\left(A \times \mathbb{H}^{2}\right) \geq \mathbf{P}[o \in \bigcup \mathcal{K}] \operatorname{area}(A) .
$$

Note that the measure $\mu^{X}\left(\mathbb{H}^{2} \times \cdot\right)$ on $\mathbb{H}^{2}$ is supported on the vertices of the Voronoi tiling that are on the outer boundaries of the empires. Let $z$ be such a vertex. Note that if $z$ does not belong to a red tile, then $z$ is in the interior of the union of two empires. In that case, $\mu^{X}\left(\mathbb{H}^{2} \times\{z\}\right)=0$, since the contributions to $\mu^{X}\left(\mathbb{H}^{2} \times\{z\}\right)$ from both empires cancel. Consequently, $\mu^{X}\left(\mathbb{H}^{2} \times \cdot\right)$ is supported on the vertices that are on the boundaries of red tiles. Suppose that $v$ is a Voronoi vertex and there are three tiles $T_{1}, T_{2}, T_{3}$ meeting at $v$, and $\alpha_{1}, \alpha_{2}, \alpha_{3}$ are their angles at $v$, respectively. Since the Voronoi tiles are convex, we have $\alpha_{1}, \alpha_{2}, \alpha_{3} \in$ $(0, \pi]$.

If $T_{1}$ is red, $T_{2}$ is white, and $T_{3}$ is black, then $\mu^{X}\left(\mathbb{H}^{2} \times\{v\}\right)=\pi-\alpha_{2}+\pi-\alpha_{3}=\alpha_{1}$ or $\mu^{X}\left(\mathbb{H}^{2} \times\{v\}\right)=0$ (the latter happens if $v \notin \partial \cup \mathcal{K}$ ). If $T_{1}$ is red, and $T_{2}$ and $T_{3}$ are both of the same color, then $\mu^{X}\left(\mathbb{H}^{2} \times\{v\}\right)=\pi-\alpha_{2}-\alpha_{3}=\alpha_{1}-\pi \leq 0$ or $\mu^{X}\left(\mathbb{H}^{2} \times\{v\}\right)=0$. If $T_{1}$ and $T_{2}$ are red, then $\mu^{X}\left(\mathbb{H}^{2} \times\{v\}\right)=\pi-\alpha_{3}=\alpha_{1}+\alpha_{2}-\pi$ 
or $\mu^{X}\left(\mathbb{H}^{2} \times\{v\}\right)=0$. If there is no red tile among $T_{1}, T_{2}, T_{3}$ or if all of them are red, then $\mu^{X}\left(\mathbb{H}^{2} \times\{v\}\right)=0$. Consequently, given the tiling (but not the colors), the expected value of $\mu^{X}\left(\mathbb{H}^{2} \times\{v\}\right)$ is bounded by

$$
2 p_{R} p_{B} p_{W}\left(\alpha_{1}+\alpha_{2}+\alpha_{3}\right)+p_{R}^{2}\left(1-p_{R}\right)\left(2 \alpha_{1}+2 \alpha_{2}+2 \alpha_{3}-3 \pi\right)=2 \pi p(1-2 p) .
$$

Hence,

$$
\mu\left(\mathbb{H}^{2} \times A\right) \leq D_{\vee} 2 \pi p(1-2 p) \operatorname{area}(A) .
$$

Using the Mass Transport Principle and (6.2), this gives

$$
\mathbf{P}[o \in \bigcup \mathcal{K}] \leq D_{\vee} 2 \pi p(1-2 p) \text {. }
$$

We now show

$$
\mathbf{P}[o \in \bigcup \mathcal{K}] \geq p_{B}+p_{W}=2 p
$$

For this, we prove that every white or black tile is contained in the hull of an empire. If not, there is a sequence of black or white clusters $K_{1}, K_{2}, \ldots$ such that each $K_{j}$ is contained in the hull of $K_{j+1}$. If infinitely many of these clusters are white, say, then when all the red tiles are changed to black, there is still no unbounded black cluster. However, $p_{R}+p_{B}>1 / 2 \geq p_{c}(\lambda)$, which is a contradiction. A similar contradiction is obtained if infinitely many of the clusters $K_{j}$ are black. Hence (6.4) holds.

Since $D_{\mathrm{F}}=\lambda$, by Remark 6.1, combining (6.3), (6.4), and (5.3) gives

$$
(2 \lambda \pi+1)(1-2 p) \geq 1 \text {. }
$$

This inequality must be satisfied for every $p<p_{c}(\lambda)$, which proves the theorem.

\section{Lemma 6.5.}

$$
\forall \lambda>0, \quad p_{c}(\lambda)>0 .
$$

Proof. Let $Q$ be a set of points in the hyperbolic plane which is maximal with the property that the distance between any two points in $Q$ is at least 1 . Then every open ball of radius 1 contains a point in $Q$.

Consider Voronoi percolation with some parameters $(p, \lambda)$. As always, let $W$ and $B$ denote the Poisson point processes of the white and black nuclei, respectively. Fix some large $R>0$. Given $y \in \mathbb{H}^{2}$, consider the Voronoi tiling $T(y)$ with nuclei $W \cup\{y\} \cup B$, and let $\widehat{W}(y)$ denote the union of the tiles of $T(y)$ with nuclei in $W \cup\{y\}$. Let $\mathcal{A}(y)$ be the event that some component of $\widehat{W}(y)$ intersects the hyperbolic circle of radius $R$ with center $y$ and the hyperbolic circle of radius 1 with center $y$. The reason for introducing $T(y)$ and $\widehat{W}(y)$ is that the events $\mathcal{A}(y)$ and $\mathcal{A}\left(y^{\prime}\right)$ are independent when $\operatorname{dist}\left(y, y^{\prime}\right)>4 R$, because $\mathcal{A}(y)$ depends only on the intersections of $B$ and $W$ with the closed ball of radius $2 R$ about $y$. The analogous property does not hold for $\widehat{W}$ in place of $\widehat{W}(y)$. (That is, the event that there is a component of $\widehat{W}$ which intersects the circles of radii 1 and $R$ about $y$ is not independent from the corresponding event for $y^{\prime}$, even if the distance between $y$ and $y^{\prime}$ is large.)

Let $\alpha(R)$ be the probability that the tile $S$ with nucleus $y$ in $T(y)$ intersects the circle of radius $R$ about $y$. We now estimate $\alpha(R)$ from above. Indeed, if $S$ intersects this circle at a point $z$, then the open ball of radius $R$ about $z$ does not contain any point in $B \cup W$. Then there is a point $x \in Q$ with $\operatorname{dist}(x, z)<1$ such 
that the ball $B(x, R-1)$ does not intersect $B \cup W$. For a given $x$, the probability for that is $\exp (-\lambda$ area $(B(x, R-1)))$, which is less than $\exp \left(-c \lambda e^{R}\right)$ for some constant $c>0$ and all $R$ sufficiently large (recall that area $(B(x, R)) / e^{R}$ tends to a positive constant as $R \rightarrow \infty)$. Consequently,

$$
\begin{aligned}
\alpha(R) & \leq|B(y, R+1) \cap Q| \exp \left(-c \lambda e^{R}\right) \leq O(1) \operatorname{area}(B(y, R+1)) \exp \left(-c \lambda e^{R}\right) \\
& \leq O(1) \exp \left(-c^{\prime} \lambda e^{R}\right),
\end{aligned}
$$

for large $R$. Clearly, $\mathbf{P}[\mathcal{A}(y)] \geq \alpha(R)$. However, there is some very small $p(R)>0$ such that $\mathbf{P}[\mathcal{A}(y)] \leq 2 \alpha(R)$ if $p<p(R)$.

Assume that $p<p(R)$. Let $o \in \mathbb{H}^{2}$ be some basepoint, and let $\widehat{W}_{o}$ be the component of $o$ in $\widehat{W}$ (set $\widehat{W}_{o}=\varnothing$ if $o \notin \widehat{W}$ ). Assuming that $\widehat{W}_{o}$ is unbounded, there is a.s. a path $\gamma:[0, \infty) \rightarrow \widehat{W}_{o}$ starting at $\gamma(0)=o$ and with $\operatorname{dist}(\gamma(t), o) \rightarrow \infty$ as $t \rightarrow \infty$. In this case, let $t_{0}:=0, y_{0}:=o$, and inductively set $t_{n}:=\sup \{t$ : $\left.\operatorname{dist}\left(\gamma(t), y_{n-1}\right)=5 R\right\}$ and $y_{n}:=\gamma\left(t_{n}\right)$. Let $y_{n}^{\prime}$ be a point in $Q$ closest to $y_{n}$. Then for each $n$, dist $\left(y_{n}^{\prime}, y_{n+1}^{\prime}\right) \leq 5 R+2$, and for each $j \neq k$, dist $\left(y_{k}^{\prime}, y_{j}^{\prime}\right) \geq 5 R-2$. Observe that the events $\mathcal{A}\left(y_{k}^{\prime}\right)$ all hold. Consequently, for every $n=1,2, \ldots$, we may bound the event that $o$ is in an unbounded component of $\widehat{W}$ by

$$
\sum_{x_{0}, x_{1}, \ldots, x_{n}} \mathbf{P}\left[\mathcal{A}\left(x_{0}\right) \cap \mathcal{A}\left(x_{1}\right) \cap \cdots \cap \mathcal{A}\left(x_{n}\right)\right],
$$

where the sum is over all sequences $x_{0}, x_{1}, \ldots, x_{n}$ in $Q$ such that $x_{0}$ is within distance 1 of $o$, each $x_{j}$ is within distance $5 R+2$ of $x_{j-1}$, and $\operatorname{dist}\left(x_{j}, x_{k}\right) \geq$ $5 R-2$ when $j \neq k$. Assuming $R>2$, these events $\mathcal{A}\left(x_{0}\right), \mathcal{A}\left(x_{1}\right), \ldots, \mathcal{A}\left(x_{n}\right)$ are independent, and we get the bound

$$
\mathbf{P}\left[\widehat{W}_{o} \text { is unbounded }\right] \leq(2 \alpha(R))^{n+1} \mid\left\{\text { such sequences } x_{0}, \ldots, x_{n}\right\} \mid .
$$

Now, the number of such sequences is at most

$$
\max \left\{|Q \cap B(z, 5 R+2)|^{n}: z \in \mathbb{H}^{2}\right\}
$$

times the number of possible choices of $x_{0}$. This is at most $\exp \left(c_{1} R n\right)$, for some constant $c_{1}$. By our estimate (6.5) for $\alpha(R)$, it is clear that there is some large $R_{0}>0$ such that the right hand side of (6.6) goes to zero as $n \rightarrow \infty$. Then for $p<$ $p\left(R_{0}\right)$ the probability that $\widehat{W}_{o}$ is unbounded is zero, and hence $p_{c}(\lambda) \geq p\left(R_{0}\right)$.

Lemma 6.6. $p_{c}(\lambda)$ is continuous on $(0, \infty)$.

Proof. Note that given $\lambda, h>0$, a union of two independent Poisson point processes with intensities $\lambda$ and $h$ is a Poisson point process with intensity $\lambda+h$. Fix some $o \in \mathbb{H}^{2}$, and let $\theta$ be the probability that $o$ is in an unbounded component of $\widehat{W}$. Consider $\theta=\theta\left(\lambda_{W}, \lambda_{B}\right)$ as a function of $\lambda_{W}=p \lambda$ and $\lambda_{B}=(1-p) \lambda$. It is clear that $\theta$ is monotone increasing (weakly) in $\lambda_{W}$ and monotone decreasing (weakly) in $\lambda_{B}$. Consequently, if $\lambda^{\prime}>\lambda$, we must have $p_{c}\left(\lambda^{\prime}\right) \lambda^{\prime} \geq p_{c}(\lambda) \lambda$ and $\left(1-p_{c}\left(\lambda^{\prime}\right)\right) \lambda^{\prime} \geq\left(1-p_{c}(\lambda)\right) \lambda$. Hence,

$$
p_{c}(\lambda) \frac{\lambda}{\lambda^{\prime}} \leq p_{c}\left(\lambda^{\prime}\right) \leq 1-\left(1-p_{c}(\lambda)\right) \frac{\lambda}{\lambda^{\prime}},
$$

which implies continuity. 
Proof of Theorem 1.5. Part (a) follows from Theorem 6.4 and Lemma 6.5. Part (b) follows from (a). Part (c) is Lemma 6.6, above. The proof from [BLPS99b] of Theorem 1.3 can easily be adapted to prove $(\mathrm{d})$.

\section{Open PROBLEMS}

Question 7.1. In the absence of planarity, most of the proofs in this paper are invalid. Which results can be extended to transitive graphs that are quasi-isometric to the hyperbolic plane, but are not planar?

Conjecture 7.2. $\lim _{\lambda \rightarrow \infty} p_{c}(\lambda)=1 / 2$.

Question 7.3. What are the asymptotics of $p_{c}(\lambda)$ as $\lambda \rightarrow \infty$ and as $\lambda \rightarrow 0$ ? In particular, what is $p_{c}^{\prime}(0)$ ?

Conjecture 7.4. $p_{c}(\lambda)$ is strictly monotone increasing.

Given $a>0$, let $a \mathbb{H}^{2}$ denote $\mathbb{H}^{2}$ with the metric scaled by $a$. It is clear that $(p, \lambda)$-Voronoi percolation on $\mathbb{H}^{2}$ is the same as $(p, 1)$-Voronoi percolation on $\sqrt{\lambda} \mathbb{H}^{2}$. As $a \rightarrow \infty$, the space $a \mathbb{H}^{2}$ looks more and more like $\mathbb{R}^{2}$ ( $a \mathbb{H}^{2}$ has constant curvature $\left.-1 / a^{2}\right)$. This leads to the following question.

Question 7.5. It is known that for Voronoi percolation in the Euclidean plane $p_{c} \geq 1 / 2$ [Zva96] (in the Euclidean setting, $p_{c}$ clearly does not depend on $\lambda$ ). However, the conjecture $p_{c}=1 / 2$ is still open. Lacking is a proof that there are unbounded components at $p>1 / 2$. Is it possible to prove that $p_{c}=1 / 2$ in the Euclidean setting, by taking a limit as $\lambda \rightarrow \infty$ in the hyperbolic setting?

This direction can also lead to a plausible guess as to the asymptotics of $p_{c}(\lambda)$ as $\lambda \rightarrow \infty$. Recall the notion of the correlation length $\xi(p)$ (see [Gri89]), which roughly measures the length scale at which $\operatorname{Bernoulli}(p)$ percolation is substantially different from Bernoulli $\left(p_{c}\right)$ percolation. Similarly, at lengths on the order of $a$ or higher, the space $a \mathbb{H}^{2}$ appears substantially different from $\mathbb{R}^{2}$. On balls of size smaller than $a,(p, 1)$-Voronoi percolation on $a \mathbb{H}^{2}$ does not look too different from $(p, 1)$-Voronoi percolation on $\mathbb{R}^{2}$. This suggests that if $\xi(p)$ is significantly smaller than $a$ (here $\xi(p)$ is the correlation length for $(p, 1)$-Voronoi percolation on $\left.\mathbb{R}^{2}\right)$, then there will be no unbounded white clusters for $(p, 1)$-Voronoi percolation on $a \mathbb{H}^{2}$. Conversely, if $\xi(p)$ is significantly larger than $a$, the hyperbolicity of $a \mathbb{H}^{2}$ appearing on the scale of $a$ will help to create unbounded white clusters. Since $(p, 1)$-Voronoi percolation on $\sqrt{\lambda} \mathbb{H}^{2}$ is the same as $(p, \lambda)$-Voronoi percolation on $\mathbb{H}^{2}$, this suggests that $\xi\left(p_{c}(\lambda)\right)$ should grow roughly at the same rate as $\sqrt{\lambda}$ when $\lambda \rightarrow \infty$. It is conjectured that $\xi(p)$ grows like $\left|p-p_{c}\right|^{-4 / 3}$, which leads to the guess that $p_{c}(\lambda)$ is asymptotic to $\frac{1}{2}-\lambda^{-2 / 3}$.

\section{ACKNOWLEDGEMENT}

We wish to express gratitude to Harry Kesten, Russ Lyons, Bojan Mohar, Igor Pak, Yuval Peres, Roberto Schonmann, and Bill Thurston for fruitful conversations and useful advice.

\section{REFERENCES}

[Bab97] L. Babai, The growth rate of vertex-transitive planar graphs, in Proceedings of the Eighth Annual ACM-SIAM Symposium on Discrete Algorithms (New Orleans, LA, 1997), ACM, New York, 1997, pp. 564-573. MR 97k:68011 
[BK89] R. M. Burton and M. Keane, Density and uniqueness in percolation, Comm. Math. Phys. 121 (1989), no. 3, 501-505. MR 90g:60090

[BK91] Topological and metric properties of infinite clusters in stationary twodimensional site percolation, Israel J. Math. 76 (1991), no. 3, 299-316. MR 93i:60182

[BLPS99a] I. Benjamini, R. Lyons, Y. Peres, and O. Schramm, Group-invariant percolation on graphs, Geom. Funct. Anal. 9 (1999), no. 1, 29-66. MR 99m:60149

[BLPS99b] Critical percolation on any nonamenable group has no infinite clusters, Ann. Probab. 27 (1999), no. 3, 1347-1356. MR 2000k:60197

[BLPS00] U Uniform spanning forests, Ann. Probab. (2000), to appear.

[BLS99] I. Benjamini, R. Lyons, and O. Schramm, Percolation perturbations in potential theory and random walks, in M. Picardello and W. Woess, editors, Random Walks and Discrete Potential Theory, Sympos. Math., Cambridge University Press, Cambridge, 1999, pp. 56-84. Papers from the workshop held in Cortona, 1997.

[BS96] I. Benjamini and O. Schramm, Percolation beyond $\mathbb{Z}^{d}$, many questions and a few answers, Electron. Comm. Probab. 1 (1996), no. 8, 71-82 (electronic). MR 97j:60179

[BS99] Recent progress on percolation beyond $\mathbb{Z}^{d}$, http://www.wisdom.weizmann.ac.il/ schramm/papers/pyond-rep/, 1999.

[BSt90] A. F. Beardon and K. Stephenson, The uniformization theorem for circle packings, Indiana Univ. Math. J. 39 (1990), no. 4, 1383-1425. MR 92b:52038

[CFKP97] J. W. Cannon, W. J. Floyd, R. Kenyon, and W. R. Parry, Hyperbolic geometry, in Flavors of Geometry, Cambridge University Press, Cambridge, 1997, pp. 59-115. MR 99c:57036

[DS84] P. G. Doyle and J. L. Snell, Random walks and electric networks, Mathematical Association of America, Washington, DC, 1984. MR 89a:94023

[GN90] G. R. Grimmett and C. M. Newman, Percolation in $\infty+1$ dimensions, in G. R. Grimmett and D. J. A. Welsh, editors, Disorder in Physical Systems, Oxford University Press, New York, 1990, pp. 167-190. MR 92a:60207

[Gri89] G. Grimmett, Percolation, Springer-Verlag, New York, 1989. MR 90j:60109

[Häg97] O. Häggström, Infinite clusters in dependent automorphism invariant percolation on trees, Ann. Probab. 25 (1997), no. 3, 1423-1436. MR 98f:60207

[HP99] O. Häggström and Y. Peres, Monotonicity of uniqueness for percolation on Cayley graphs: all infinite clusters are born simultaneously, Probab. Theory Related Fields 113 (1999), no. 2, 273-285. MR 99k:60253

[HS95] Z.-X. He and O. Schramm, Hyperbolic and parabolic packings, Discrete Comput. Geom. 14 (1995), no. 2, 123-149. MR 96h:52017

[Imr75] W. Imrich, On Whitney's theorem on the unique embeddability of 3-connected planar graphs, in Recent Advances in Graph Theory (Proc. Second Czechoslovak Sympos., Prague, 1974), Academia, Prague, 1975, pp. 303-306. MR 52:5462

[Lal98] S. P. Lalley, Percolation on Fuchsian groups, Ann. Inst. H. Poincaré Probab. Statist. 34 (1998), no. 2, 151-177. MR 99g:60190

[Lyo00] R. Lyons, Phase transitions on nonamenable graphs, J. Math. Phys. 41 (2000), no. 3, 1099-1126. Probabilistic techniques in equilibrium and nonequilibrium statistical physics. CMP 2000:13

[Lyo01] _ Probability on trees and networks, Cambridge University Press, 2001. Written with the assistance of $\mathrm{Y}$. Peres, in preparation. Current version available at http://php. indiana.edu/ rdlyons/.

[Mad70] W. Mader, Über den Zusammenhang symmetrischer Graphen, Arch. Math. (Basel) 21 (1970), 331-336. MR 44:6534

[MR96] R. Meester and R. Roy, Continuum percolation, Cambridge University Press, Cambridge, 1996. MR 98d:60193

[PSN00] I. Pak and T. Smirnova-Nagnibeda, On non-uniqueness of percolation on nonamenable Cayley graphs, C. R. Acad. Sci. Paris Sér. I Math. 330 (2000), no. 6, 495-500. MR 2000m:60116

[Per00] Y. Peres, Percolation on nonamenable products at the uniqueness threshold, Ann. Inst. H. Poincaré Probab. Statist. 36 (2000), no. 3, 395-406. CMP 2000:15

[Sch99a] R. H. Schonmann, Percolation in $\infty+1$ dimensions at the uniqueness threshold, in $\mathrm{M}$. Bramson and R. Durrett, editors, Perplexing Probability Problems: Papers in Honor of Harry Kesten, Birkhäuser, Boston, 1999, pp. 53-67. CMP 99:16 
[Sch99b] _ Stability of infinite clusters in supercritical percolation, Probab. Theory Related Fields 113 (1999), no. 2, 287-300. MR 99k:60252

[SS97] P. Schmutz Schaller, Extremal Riemann surfaces with a large number of systoles, in Extremal Riemann Surfaces (San Francisco, CA, 1995), Amer. Math. Soc., Providence, RI, 1997, pp. 9-19. MR 98c:11064

[Wat70] M. E. Watkins, Connectivity of transitive graphs, J. Combinatorial Theory 8 (1970), 23-29. MR 42:1707

[Zva96] A. Zvavitch, The critical probability for Voronoi percolation, MSc. thesis, Weizmann Institute of Science, 1996.

Department of Mathematics, Weizmann Institute of Science, Rehovot 76100, Israel E-mail address: itai@wisdom.weizmann.ac.il

$U R L:$ http://www.wisdom.weizmann.ac.il/ ${ }^{\text {itai/ }}$

Department of Mathematics, Weizmann Institute of Science, Rehovot 76100, Israel

E-mail address: schramm@icrosoft.com 